\title{
A full-duplex SWIPT system with self-energy recycling to minimize energy consumption
}

\author{
Hyunjong $\mathrm{Na}^{1}$ and Chungyong Lee ${ }^{2^{*}}$ (D)
}

\begin{abstract}
In simultaneous wireless information and power transfer system (SWIPT), additional resource allocation for power transfer degrades system performance. Therefore, how to allocate power and time to data transmission and energy transfer is very important for the performance of time switching-based SWTPT system. In this paper, we propose a time switching-based full-duplex SWIPT system combined with self-energy recycling since self-interference harvesting is more efficient than simultaneous data transmission if the magnitude of self-interference is sufficiently large. Proposed system has additional self-energy recycling phases compared to the conventional time switching-based full-duplex SWIPT system. Considering the limitation of maximum transmission power, proposed system is optimized transmission time as well as power. For the validity of the proposed system, we show the bi-convexity of proposed system and propose an iterative algorithm for optimization of proposed system. In simulations, we compare proposed system with the half-duplex system without simultaneous data transmission phase and the full-duplex system without self-energy recycling phase. Simulation results show proposed system consume less energy than the conventional systems.
\end{abstract}

Keywords: SWIPT, Full duplex, Device-to-device communication, Self-energy recycling

\section{Introduction}

In recent years, simultaneous wireless information and power transfer (SWIPT) schemes are attracting in terms of enabling to carry information and energy simultaneously [1]. SWIPT schemes can be classified into two categories according to receiver types: time switching (TS) SWIPT scheme and power splitting (PS) SWIPT scheme. TS SWIPT scheme divides the downlink (DL) time into energy harvesting (EH) phase and information detection (ID) phase, and PS SWIPT scheme splits the received signal into EH signal and ID signal. Since the amount of harvested energy is also important as well as the data rate in SWIPT system, the trade-off between data rate and power transfer has been studied [1, 2]. Furthermore, various optimization problems such as power minimization, energy efficiency maximization, and weighted sumrate maximization were studied in various system models [3-5].

\footnotetext{
*Correspondence: cylee@yonsei.ac.kr

${ }^{2}$ The Department of Electrical and Electronic Engineering, Yonsei University, C527, 03722 Seoul, South Korea

Full list of author information is available at the end of the article
}

SWIPT system combined with full duplex (FD) has advantages including the increase of spectral efficiency. In contrast to the conventional FD system where selfinterference (SI) is harmful, SI can be beneficial in terms of energy source for harvesting in FD SWIPT system [6-10]. A number of studies using this advantage were performed for FD relay system where relay has no external power supply except for transmitted power from base station (BS) $[7,8,10]$. Also, the self-energy recycling (S-ER) scheme was proposed in point-to-point (P2P) FD system where PS-based EH is used at user equipment (UE) [9]. In general, the performance of PS SWIPT scheme is better than that of TS SWIPT scheme, because TS SWIPT scheme can be treated as a special case of PS SWIPT scheme with binary split power ratios $[4,11]$. However, in contrast to TS receiver where a device requires only a simple switcher, PS receiver needs an RF signal splitter and its structure is relatively complicate $[4,12]$. This means that TS-based SWIPT scheme is more applicable to UE than PS-based SWIPT scheme since the structure of UE needs to be simple. 
TS-based SWIPT systems with S-ER were studied in [13-15]. In [13], Zeng and Zhang proposed the beamformer design to maximize end-to-end throughput in multiple-input single-output (MISO) relay system. In the first phase, the source transmits information to relay, and the received signal at relay is amplified and forwarded to the destination during the second phase. At the same time, the source transfers the energy to relay, and relay harvests SI as well as the signal from the source node in the second phase. Ding et al. proposed the beamforming scheme of multiple-input multiple-output (MIMO) relay for rate maximization where relay harvests SI in the second phase [14]. In [15], Hwang et al. proposed the beamformers of BS and relay where relay has additional antenna to harvest SI.

The FD SWIPT system with S-ER is also helpful to device-to-device (D2D) communication for Internet of Thing (IoT) system-the communication between mobile phone and devices which cannot have sufficient battery. In the communication between smart phone and IoT devices such as bluetooth devices and smart watch, IoT devices are not connected with BS. Also, the short lifetime of IoT devices due to small size battery is a critical problem. In this environment, the device equipped with a large battery, such as a smartphone, can transfer energy-to-energy-deficient devices to prolong communication time.

Thus, we propose a TS-based FD SWIPT system for D2D communication to prolong the lifetime by minimizing energy consumption under the target rate constraint. In contrast to relay systems, D2D communication systems need to be considered as S-ER of both users since both users are not connected to power grid. Thus, proposed system has additional S-ER phases contrary to the conventional TS-based FD SWIPT system to employ SI efficiently. The entire time in the conventional TS-based FD SWIPT system consists of two phases if one user does not have enough energy to transmit data. In the first phase, one $\mathrm{UE}$, i.e., $\mathrm{UE}_{1}$, transmits energy to another $\mathrm{UE}$, i.e., $\mathrm{UE}_{2}$, and $\mathrm{UE}_{2}$ transmits data to $\mathrm{UE}_{1}$, and data transmissions in both directions are operated in the second phase.
In the proposed system, however, each phase is divided into two parts for S-ER implementation. In these S-ER phases, $\mathrm{UE}_{2}$ does not transmit any signal and $\mathrm{UE}_{1}$ harvests SI. To minimize energy consumption, we should find time duration and power corresponding to each phase. Since the optimization problem for proposed system has a form of bi-convex, we propose an iterative scheme based on line search method to find a solution. In contrast to the alternative convex search (ACS) technique which can obtain a local minimum, the proposed scheme can obtain a better solution close to global optimum.

\section{System model and optimization problem formulation}

\subsection{System model}

As depicted in Fig. 1, we consider a D2D FD SWIPT system with the assumption that $\mathrm{UE}_{2}$ does not have energy for data transmission but $\mathrm{UE}_{1}$ has sufficient energy. In this scenario, $\mathrm{UE}_{1}$ should transfer energy to $\mathrm{UE}_{2}$ for data communication with $\mathrm{UE}_{2}$. However, since $\mathrm{UE}_{1}$ also does not connected to power grid, the total energy consumption of $\mathrm{UE}_{1}$ should be minimized. For this purpose, each user can harvest SI since harvesting SI can be more efficient than simultaneous data transmission if the magnitude of SI is sufficiently large. The proposed system model needs additional phases to harvest SI compared to the conventional TS-based FD SWIPT systems, and the operating protocol consists of four phases according to the mode of each user-energy transfer or data transmission mode of $\mathrm{UE}_{1}$ and only receive or data transmission mode of $\mathrm{UE}_{2}$. Also, it is assumed that all channels have quasi-static flat-fading characteristics, and the length of one channel block is normalized. Then, the relationship between time duration of each phase can be written as:

$$
\tau_{1}+\tau_{2}+\tau_{3}+\tau_{4}=1
$$

and the received data signals at $\mathrm{UE}_{1}$ and $\mathrm{UE}_{2}$ can be, respectively, expressed as:

$$
y_{1,2}=\sqrt{P_{2,2}} h_{12} s_{2,2}+\sqrt{P_{1,2}} g_{1} s_{1,2}+n_{1},
$$

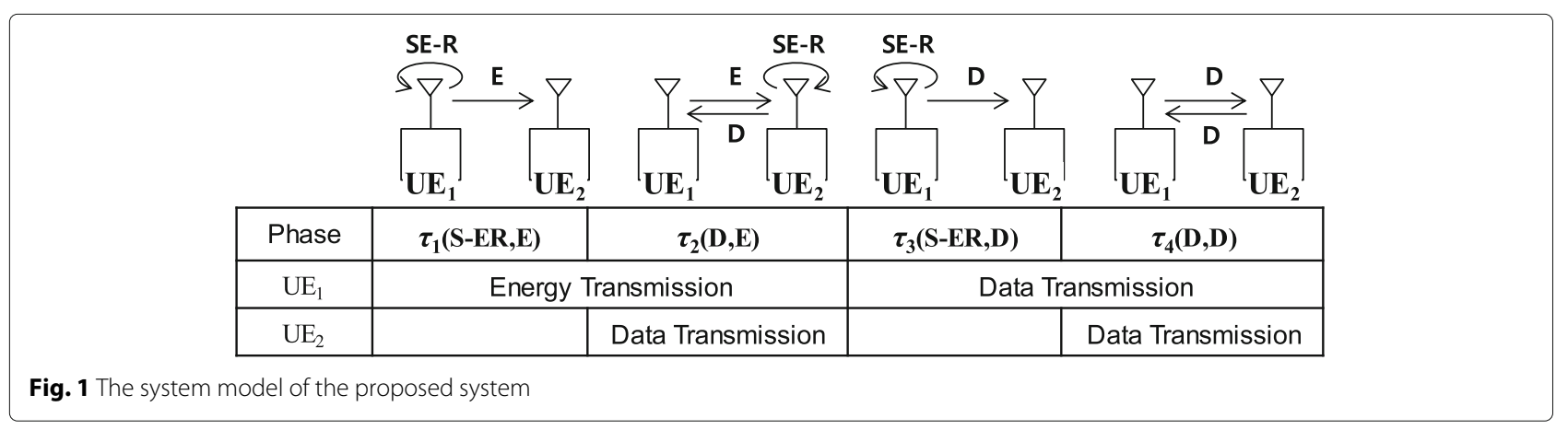




$$
\begin{aligned}
& y_{2,3}=\sqrt{P_{1,3}} h_{21} s_{1,3}+n_{2}, \\
& y_{1,4}=\sqrt{P_{2,4}} h_{12} s_{2,4}+\sqrt{P_{1,4}} g_{1} s_{1,4}+n_{1}, \\
& y_{2,4}=\sqrt{P_{1,4}} h_{21} s_{1,4}+\sqrt{P_{2,4}} g_{2} s_{2,4}+n_{2},
\end{aligned}
$$

where $y_{k, i}$ and $P_{k, i}$ mean the received signal and the transmission power at $\mathrm{UE}_{k}$ corresponding to $\tau_{i}$, respectively; $h_{i j}$ denotes the channel from $\mathrm{UE}_{j}$ to $\mathrm{UE}_{i}$ with $h_{i j} \sim$ $\mathcal{C N}\left(0, \sigma^{2}\right)$; and $g_{k}$ denotes SI channel at $\mathrm{UE}_{k}$ with a static amplitude and random phase characteristics, i.e., $\left|g_{k}\right|^{2}=$ $\sigma_{S I}^{2}[7] . s_{k, i}$ means the transmit symbol of $\mathrm{UE}_{k}$ corresponding to $\tau_{i}$, and $n_{k}$ is the additive white Gaussian noise at $\mathrm{UE}_{k}$ with $n_{k} \sim \mathcal{C} \mathcal{N}\left(0, N_{0}\right)$. In energy transmission phases of $\mathrm{UE}_{1}$, i.e., $\tau_{1}$ and $\tau_{2}, \mathrm{UE}_{2}$ harvests the energy transmitted from $U_{1}$. At the same time, since SI can be used additional energy source during $\tau_{2}, \mathrm{UE}_{2}$ harvests SI without SI cancelation. During the phases of not receiving data from $\mathrm{UE}_{2}$, i.e., $\tau_{1}$ and $\tau_{3}, \mathrm{UE}_{1}$ also harvests SI without SI cancelation. Then, the harvested energies at $\mathrm{UE}_{1}$ and $\mathrm{UE}_{2}$ can be, respectively, written as:

$$
\begin{aligned}
& E_{1,1}^{\mathrm{har}}=\tau_{1} \eta P_{1,1}\left|g_{1}\right|^{2}, \\
& E_{2,1}^{\mathrm{har}}=\tau_{1} \eta P_{1,1}\left|h_{21}\right|^{2}, \\
& E_{2,2}^{\mathrm{har}}=\tau_{2} \eta\left(P_{1,2}\left|h_{21}\right|^{2}+P_{2,2}\left|g_{2}\right|^{2}\right), \\
& E_{1,3}^{\mathrm{har}}=\tau_{3} \eta P_{1,3}\left|g_{1}\right|^{2},
\end{aligned}
$$

where $E_{k, i}^{\mathrm{har}}$ is the harvested energy at $\mathrm{UE}_{k}$ during $\tau_{i}$, and $\eta$ means power conversion efficiency.

\subsection{Optimization problem for the proposed system}

The system energy consumption is the same with the total energy consumption of $\mathrm{UE}_{1}$. Thus, the system energy consumption, $E_{T}$, can be written as:

$$
E_{T}=\tau_{1}\left(1-\eta \sigma_{\mathrm{SI}}^{2}\right) P_{1,1}+\tau_{2} P_{1,2}+\tau_{3}\left(1-\eta \sigma_{\mathrm{SI}}^{2}\right) P_{1,3}+\tau_{4} P_{1,4}
$$

Since total energy consumption of $\mathrm{UE}_{2}$ should not be larger than the sum of the energies harvested from $\mathrm{UE}_{1}$ and SI, the relationship between the powers of $\mathrm{UE}_{1}$ and $\mathrm{UE}_{2}$ can be expressed as:

$$
\eta\left|h_{21}\right|^{2}\left(\tau_{1} P_{1,1}+\tau_{2} P_{1,2}\right) \geq \tau_{2}\left(1-\eta \sigma_{\text {SI }}^{2}\right) P_{2,2}+\tau_{4} P_{2,4}
$$

Assuming that SI is imperfectly canceled in data receiving phase [16], the achievable rate of each user can be, respectively, written as:

$$
\begin{array}{r}
R_{1}=\tau_{2} \log _{2}\left(1+\frac{\left|h_{12}\right|}{N_{0}+\sigma_{\mathrm{RSI}}^{2}} P_{2,2}\right) \\
+\tau_{4} \log _{2}\left(1+\frac{\left|h_{12}\right|}{N_{0}+\sigma_{\mathrm{RSI}}^{2}} P_{2,4}\right) \\
R_{2}=\tau_{3} \log _{2}\left(1+\frac{\left|h_{21}\right|}{N_{0}} P_{1,3}\right) \\
\quad+\tau_{4} \log _{2}\left(1+\frac{\left|h_{21}\right|}{N_{0}+\sigma_{\mathrm{RSI}}^{2}} P_{1,4}\right)
\end{array}
$$

where $\sigma_{\mathrm{RSI}}^{2}$ represents the variance of residual self-interference. The residual self-interference model can be divided into two cases: the complicated case and the optimistic case [17-22]. In the complicated case, the power of residual self-interference is increased with the transmission power $[17,19]$. In the optimistic case, the power of residual self-interference has constant value regardless of the transmission power [18, 20-22]. We assume that the power of residual self-interference is constant as the optimistic case. Instead, we set the power of residual self-interference to upper bound of the power of residual self-interference, i.e., $\sigma_{\mathrm{RSI}}^{2}=\sigma_{\mathrm{SIC}}^{2} \sigma_{S I}^{2} P_{\max }$ where $P_{\max }$ is the maximum transmission power of UE. Since the data transmission of $\mathrm{UE}_{2}$ does not exist during $\tau_{3}$, there is no residual self-interference during $\tau_{3}$. Then, the optimization problem to minimize the system energy consumption can be formulated as follows:

$$
\begin{aligned}
& \min _{\mathbf{P}_{1}, \boldsymbol{\tau}} E_{T} \\
& \text { s.t. } \quad \tau_{1}+\tau_{2}+\tau_{3}+\tau_{4}=1, \\
& R_{k} \geq C_{k}, \\
& \eta\left|h_{D}\right|^{2}\left(\left(1-\sigma_{\mathrm{SI}}^{2}\right) \tau_{1} P_{1,1}+\tau_{2} P_{1,2}\right) \\
& \quad \geq \tau_{2}\left(1-\eta \sigma_{\mathrm{SI}}^{2}\right) P_{2,2}+\tau_{4} P_{2,4}, \\
& \boldsymbol{\tau} \geq \mathbf{0}, \\
& 0 \leq P_{k, i} \leq P_{\max },
\end{aligned}
$$

where $C_{k}$ means a target rate of $\mathrm{UE}_{k}$. For convenience, we set $\mathbf{P}_{1}=\left[P_{1,1}, P_{1,2}, P_{1,3}, P_{1,4}\right]^{T}, \mathbf{P}_{2}=\left[0, P_{2,2}, 0, P_{2,4}\right]^{T}$, and $\boldsymbol{\tau}=\left[\tau_{1}, \tau_{2}, \tau_{3}, \tau_{4}\right]^{T}$. The optimization problem is a form of linear programming to $\tau$ when the powers of both users are fixed, and the objective function and all constraint functions are convex about $\mathbf{P}$ when $\boldsymbol{\tau}$ is fixed. Therefore, the optimization problem is a bi-convex problem [23]. However, ACS technique which can be used to solve a bi-convex optimization problem can obtain a local minimum. Thus, we propose an iterative scheme to derive the value close to the global optimum of the proposed system in the next section, and the algorithm of the iterative scheme is given in Table 1. 
Table 1 Algorithm of the proposed iterative scheme for the optimization of the proposed system

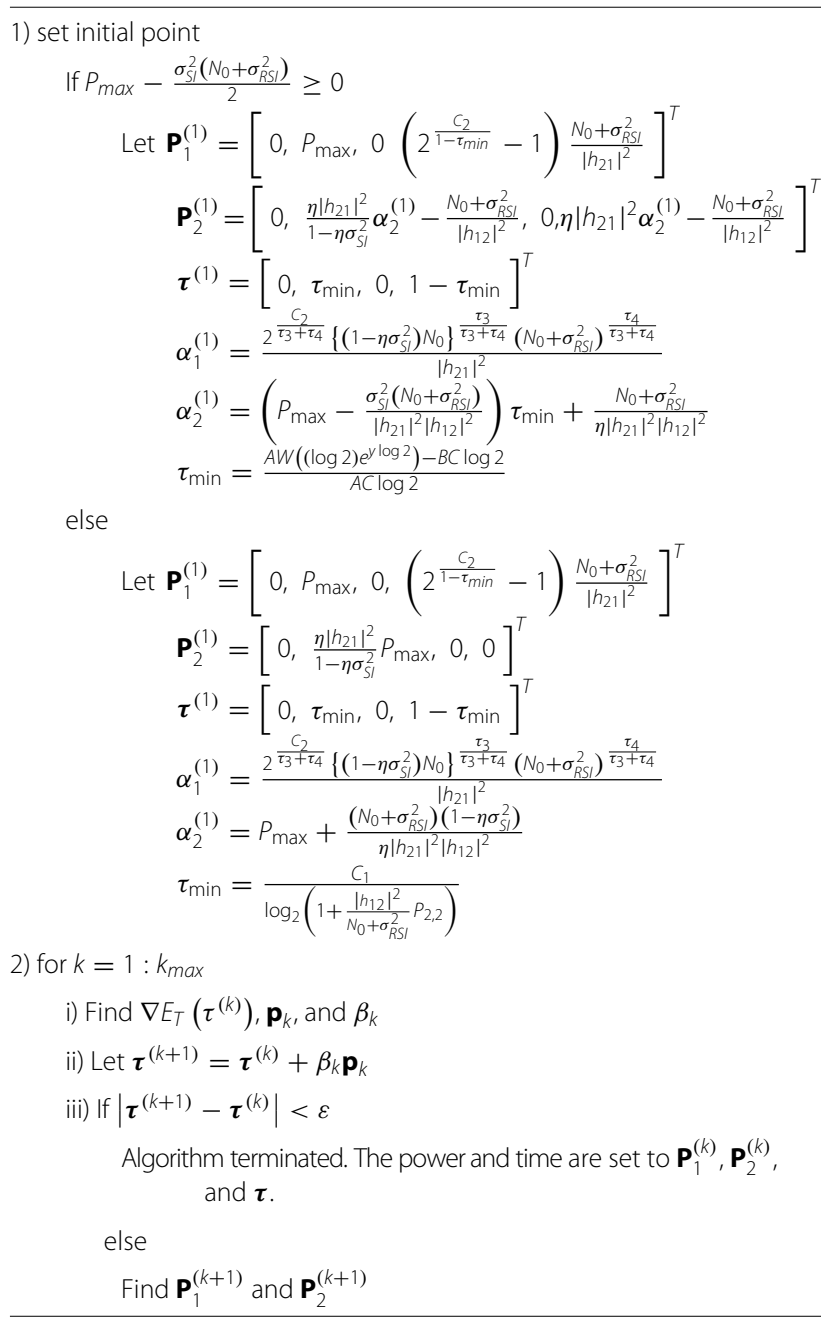

\section{Power and time optimization to minimize energy consumption of the proposed system}

\subsection{Optimal power allocation with fixed $\tau$}

Since $P_{1,1}$ and $P_{1,2}$ only affect $R_{1}$, and $P_{1,3}$ and $P_{1,4}$ only affect $R_{2}$, the optimization problem can be divided into two parts: $U_{1}$ target rate part and $U_{2}$ target rate part. Then, the optimization problem for $\mathrm{UE}_{1}$ target rate can be written as:

$$
\begin{gathered}
\min _{\mathbf{P}_{1}, \mathbf{P}_{2}} \tau_{1}\left(1-\eta \sigma_{\mathrm{SI}}^{2}\right) P_{1,1}+\tau_{2} P_{1,2} \\
\text { s.t. } \quad \tau_{1} P_{1,1}+\tau_{2} P_{1,2} \geq \tau_{2} \frac{\left(1-\eta \sigma_{\mathrm{SI}}^{2}\right)}{\eta\left|h_{21}\right|^{2}} P_{2,2}+\tau_{4} \frac{1}{\eta\left|h_{21}\right|^{2}} P_{2,4}, \\
\tau_{2} \log _{2}\left(1+\frac{P_{2,2}\left|h_{12}\right|^{2}}{N_{0}+\sigma_{\mathrm{RSI}}^{2}}\right)+\tau_{4} \log _{2}\left(1+\frac{P_{2,4}\left|h_{12}\right|^{2}}{N_{0}+\sigma_{\mathrm{RSI}}^{2}}\right) \geq C_{1} .
\end{gathered}
$$

Since $\mathrm{UE}_{1}$ only needs to transmit energy as much as the required energy of $\mathrm{UE}_{2}$ to satisfy $C_{1}$, we can divide this optimization problem into two stages. First, the minimum energy consumption of $\mathrm{UE}_{2}$ is derived to satisfy $C_{1}$ under the assumption that the energy harvested from $\mathrm{UE}_{1}$ is more than the minimum energy consumption of $\mathrm{UE}_{2}$. Next, the minimum transfer energy of $\mathrm{UE}_{1}$ is obtained based on the minimum energy consumption of $\mathrm{UE}_{2}$. Then, the optimization problem of the first stage can be expressed as:

$$
\begin{aligned}
\min _{\mathbf{P}_{2}} \tau_{2} \frac{\left(1-\eta \sigma_{\mathrm{SI}}^{2}\right)}{\eta\left|h_{21}\right|^{2}} P_{2,2}+\tau_{4} \frac{1}{\eta\left|h_{21}\right|^{2}} P_{2,4} \\
\text { s.t. } \quad \tau_{2} \log _{2}\left(1+\frac{P_{2,2}\left|h_{12}\right|^{2}}{N_{0}+\sigma_{\mathrm{RSI}}^{2}}\right) \\
\quad+\tau_{4} \log _{2}\left(1+\frac{P_{2,4}\left|h_{12}\right|^{2}}{N_{0}+\sigma_{\mathrm{RSI}}^{2}}\right) \geq C_{1} .
\end{aligned}
$$

If the objective function and all constraints are convex and the optimization problem has one possible set to satisfy the KKT conditions, then the feasible set is a global minimizer [24]. According to the KKT conditions, we can obtain following equations:

$$
\tau_{2} \frac{\left(1-\eta \sigma_{\mathrm{SI}}^{2}\right)}{\eta\left|h_{21}\right|^{2}}-\mu_{1}\left(\tau_{2} \frac{\left|h_{12}\right|^{2}}{\left(N_{0}+\sigma_{\mathrm{RSI}}^{2}+\left|h_{12}\right|^{2} P_{2,2}\right) \log 2}\right)=0,
$$

$$
\tau_{4} \frac{1}{\eta\left|h_{21}\right|^{2}}-\mu_{1}\left(\tau_{4} \frac{\left|h_{12}\right|^{2}}{\left(N_{0}+\sigma_{\mathrm{RSI}}^{2}+\left|h_{12}\right|^{2} P_{2,4}\right) \log 2}\right)=0,
$$

$$
\begin{aligned}
& \mu_{1}\left(C_{1}-\tau_{2} \log _{2}\left(1+\frac{P_{2,2}\left|h_{12}\right|^{2}}{N_{0}+\sigma_{\mathrm{RSI}}^{2}}\right)\right. \\
& \left.-\tau_{4} \log _{2}\left(1+\frac{P_{2,4}\left|h_{12}\right|^{2}}{N_{0}+\sigma_{\mathrm{RSI}}^{2}}\right)\right)=0,
\end{aligned}
$$

$$
\begin{gathered}
\mu_{1} \geq 0 \\
C_{1}-\tau_{2} \log _{2}\left(1+\frac{P_{2,2}\left|h_{12}\right|^{2}}{N_{0}+\sigma_{\mathrm{RSI}}^{2}}\right)-\tau_{4} \log _{2}\left(1+\frac{P_{2,4}\left|h_{12}\right|^{2}}{N_{0}+\sigma_{\mathrm{RSI}}^{2}}\right) \leq 0,
\end{gathered}
$$

where $\mu_{1} \neq 0$ since (17a) and (17b) are not satisfied when $\mu_{1}=0$. Then, $P_{2,2}$ and $P_{2,4}$ can be expressed in terms of $\mu_{1}$ :

$$
\begin{aligned}
& P_{2,2}^{*}=\frac{\eta\left|h_{21}\right|^{2}}{1-\eta \sigma_{\mathrm{SI}}^{2}} \alpha_{2}-\frac{N_{0}+\sigma_{\mathrm{RSI}}^{2}}{\left|h_{12}\right|^{2}}, \\
& P_{2,4}^{*}=\eta\left|h_{21}\right|^{2} \alpha_{2}-\frac{N_{0}+\sigma_{\mathrm{RSI}}^{2}}{\left|h_{12}\right|^{2}},
\end{aligned}
$$

where $\alpha_{2}=\frac{\mu_{1}^{*}}{\log 2}$ and $\mu_{1}^{*}$ can be obtained by (17c), (18), and (19) as follow: 


$$
\mu_{1}^{*}=2^{\frac{C_{1}}{\tau_{2}+\tau_{4}}}\left(1-\eta \sigma_{\mathrm{SI}}^{2}\right)^{\frac{\tau_{2}}{\tau_{2}+\tau_{4}}}\left(\frac{\left(N_{0}+\sigma_{\mathrm{RSI}}^{2}\right) \log 2}{\eta\left|h_{21}\right|^{2}\left|h_{12}\right|^{2}}\right) .
$$

Since the minimum energy consumption of $\mathrm{UE}_{2}$ is obtained under the condition of (15c), (15a) can be solved by only considering (15b). To minimize the energy consumption of $\mathrm{UE}_{1}$, it is efficient that required energy of $\mathrm{UE}_{2}$ is harvested during $\tau_{1}$ since $\tau_{1}$ is more efficient than $\tau_{2}$ due to $\mathrm{S}$-ER. However, since the maximum transmission power is limited, all required energy of $\mathrm{UE}_{2}$ may not be harvested during $\tau_{1}$. In this case, additional energy harvesting should be done during $\tau_{2}$. Thus, the transmission powers of $\mathrm{UE}_{1}$ during $\tau_{1}$ and $\tau_{2}$ can be, respectively, expressed as:

$$
\begin{aligned}
& P_{1,1}^{*}=\min \left(\frac{\tau_{2}\left(1-\eta \sigma_{\mathrm{SI}}^{2}\right) P_{2,2}^{*}+\tau_{4} P_{2,4}^{*}}{\tau_{1} \eta\left|h_{21}\right|^{2}}, P_{\max }\right), \quad \text { (21) } \\
& P_{1,2}^{*}=\frac{1}{\tau_{2}}\left(\tau_{2} \frac{\left(1-\eta \sigma_{\mathrm{SI}}^{2}\right)}{\eta\left|h_{21}\right|^{2}} P_{2,2}^{*}+\tau_{4} \frac{1}{\eta\left|h_{21}\right|^{2}} P_{2,4}^{*}-\tau_{1} P_{1,1}^{*}\right) .
\end{aligned}
$$

Then, the optimization problem for $\mathrm{UE}_{2}$ target rate can be written as:

$$
\begin{aligned}
& \min _{\mathbf{P}_{1}} \tau_{3}\left(1-\eta \sigma_{\mathrm{SI}}^{2}\right) P_{1,3}+\tau_{4} P_{1,4} \\
& \text { s.t. } \quad \tau_{3} \log _{2}\left(1+\frac{P_{1,3}\left|h_{21}\right|^{2}}{N_{0}}\right) \\
& +\tau_{4} \log _{2}\left(1+\frac{P_{1,4}\left|h_{21}\right|^{2}}{N_{0}+\sigma_{\mathrm{RSI}}^{2}}\right) \geq C_{2},
\end{aligned}
$$

using the KKT conditions, $P_{1,3}$ and $P_{1,4}$ can be expressed as:

$$
\begin{aligned}
& P_{1,3}^{*}=\frac{1}{1-\eta \sigma_{\mathrm{SI}}^{2}} \alpha_{1}-\frac{N_{0}}{\left|h_{21}\right|^{2}}, \\
& P_{1,4}^{*}=\alpha_{1}-\frac{N_{0}+\sigma_{\mathrm{RSI}}^{2}}{\left|h_{21}\right|^{2}},
\end{aligned}
$$

where $\alpha_{1}=\frac{2^{\frac{C_{2}}{\tau_{3}+\tau_{4}}}\left\{\left(1-\eta \sigma_{\mathrm{SI}}^{2}\right) N_{0}\right\}^{\frac{\tau_{3}}{\tau_{3}+\tau_{4}}}\left(N_{0}+\sigma_{\mathrm{RSI}}^{2}\right)^{\frac{\tau_{4}}{\tau_{3}+\tau_{4}}}}{\left|h_{21}\right|^{2}}$. Therefore, if $\boldsymbol{\tau}$ has fixed value, the optimal power allocation to minimize energy consumption can be written as:

$$
\mathbf{P}_{1}^{*}=\left[\begin{array}{c}
\min \left(\frac{\tau_{2}\left(1-\eta \sigma_{\mathrm{SI}}^{2}\right) P_{2,2}^{*}+\tau_{4} P_{2,4}^{*}}{\tau_{1} \eta\left|h_{21}\right|^{2}}, P_{\max }\right) \\
\frac{1}{\tau_{2}}\left(\tau_{2} \frac{\left(1-\eta \sigma_{\mathrm{SI}}^{2}\right)}{\eta\left|h_{21}\right|^{2}} P_{2,2}^{*}+\tau_{4} \frac{1}{\eta\left|h_{21}\right|^{2}} P_{2,4}^{*}-\tau_{1} P_{1,1}^{*}\right) \\
\frac{1}{1-\eta \sigma_{\mathrm{SI}}^{2}} \alpha_{1}-\frac{N_{0}}{\left|h_{21}\right|^{2}} \\
\alpha_{1}-\frac{N_{0}+\sigma_{\mathrm{RSI}}^{2}}{\left|h_{21}\right|^{2}}
\end{array}\right],
$$

$\mathbf{P}_{2}^{*}=\left[0, \frac{\eta\left|h_{21}\right|^{2}}{1-\eta \sigma_{\mathrm{SI}}^{2}} \alpha_{2}-\frac{N_{0}+\sigma_{\mathrm{RSI}}^{2}}{\left|h_{12}\right|^{2}}, 0, \eta\left|h_{21}\right|^{2} \alpha_{2}-\frac{N_{0}+\sigma_{\mathrm{RSI}}^{2}}{\left|h_{12}\right|^{2}}\right]^{T}$.

\subsection{Deriving minimum energy transmission time and initial point}

Since maximum transmission power is limited, $\tau_{\min }$ should be guaranteed to satisfy $C_{2}$. For finding $\tau_{\min }$, we assume that $\tau_{1}$ and $\tau_{3}$ are 0 to secure maximum data transmission time, and $P_{1,2}$ takes $P_{\max }$ since $P_{\max }$ is available maximum transmission power of $\mathrm{UE}_{1}$. Then, (11) can be modified with the assumption that $\tau_{1}=\tau_{3}=0$ and $P_{1,1}=P_{1,3}=0$ as:

$$
\eta\left|h_{21}\right|^{2} \tau_{2} P_{\max }=\tau_{2}\left(1-\eta \sigma_{\mathrm{SI}}^{2}\right) P_{2,2}+\left(1-\tau_{2}\right) P_{2,4},
$$

and $\alpha_{2}$ can be written in terms of $\tau_{2}$ by using (27):

$$
\alpha_{2}^{(1)}=\left(P_{\max }-\frac{\sigma_{\mathrm{SI}}^{2}\left(N_{0}+\sigma_{\mathrm{RSI}}^{2}\right)}{\left|h_{21}\right|^{2}\left|h_{12}\right|^{2}}\right) \tau_{\min }+\frac{N_{0}+\sigma_{\mathrm{RSI}}^{2}}{\eta\left|h_{21}\right|^{2}\left|h_{12}\right|^{2}} .
$$

The initial point can be derived as two cases according to the value of $A$ where $A=P_{\max }-\frac{\sigma_{\mathrm{SI}}^{2}\left(N_{0}+\sigma_{\mathrm{RSS}}^{2}\right)}{\left|h_{21}\right|^{2}\left|h_{12}\right|^{2}}$, because $A<0$ means that $P_{2,4}$ obtained by (27) is negative value.

\subsubsection{Case I: $A \geq 0$}

We can find $\tau_{\min }$ by using (17d), (27), and (29):

$$
\tau_{\min }=\frac{A W\left((\log 2) e^{y \log 2}\right)-B C \log 2}{A C \log 2}
$$

where $B=\frac{N_{0}+\sigma_{\mathrm{RSI}}^{2}}{\eta\left|h_{21}\right|^{2}\left|h_{12}\right|^{2}}, C=\log _{2}\left(\frac{1}{1-\eta \sigma_{\mathrm{SI}}^{2}}\right), D=$ $\log _{2}\left(\frac{\eta\left|h_{21}\right|^{2}\left|h_{12}\right|^{2}}{N_{0}+\sigma_{\text {RSI }}^{2}}\right)-C_{1}, y=\log _{2} \frac{C}{A}+\frac{B C}{A}-D$, and $W(\bullet)$ means Lambert $W$ function. Then, we can derive the initial point based on $\tau_{\min }$ as follows:

$$
\begin{aligned}
& \boldsymbol{\tau}^{(1)}=\left[0, \tau_{\min }, 0,1-\tau_{\min }\right]^{T}, \\
& \mathbf{P}_{1}^{(1)}=\left[0, P_{\max }, 0\left(2^{\frac{C_{2}}{1-\tau_{\min }}}-1\right) \frac{N_{0}+\sigma_{\mathrm{RSS}}^{2}}{\left|h_{21}\right|^{2}}\right]^{T}, \\
& \mathbf{P}_{2}^{(1)}=\left[0, \frac{\eta\left|h_{21}\right|^{2}}{1-\eta \sigma_{\mathrm{SI}}^{2}} \alpha_{2}^{(1)}-\frac{N_{0}+\sigma_{\mathrm{RSI}}^{2}}{\left|h_{12}\right|^{2}}, 0, \eta\left|h_{21}\right|^{2} \alpha_{2}^{(1)}-\frac{N_{0}+\sigma_{\mathrm{RSI}}^{2}}{\left|h_{12}\right|^{2}}\right]^{T}, \\
& \alpha_{1}^{(1)}=\frac{2^{\frac{C_{2}}{\tau_{3}+\tau_{4}}}\left\{\left(1-\eta \sigma_{\mathrm{SI}}^{2}\right) N_{0}\right\}^{\frac{\tau_{3}}{\tau_{3}+\tau_{4}}}\left(N_{0}+\sigma_{\mathrm{RSI}}^{2}\right)^{\frac{\tau_{4}}{\tau_{3}+\tau_{4}}}}{\left|h_{21}\right|^{2}}, \\
& \alpha_{2}^{(1)}=\left(P_{\max }-\frac{\sigma_{\mathrm{SI}}^{2}\left(N_{0}+\sigma_{\mathrm{RSI}}^{2}\right)}{\left|h_{21}\right|^{2}\left|h_{12}\right|^{2}}\right) \tau_{\min }+\frac{N_{0}+\sigma_{\mathrm{RSI}}^{2}}{\eta\left|h_{21}\right|^{2}\left|h_{12}\right|^{2}} .
\end{aligned}
$$




\subsubsection{Case II: $A<0$}

$A<0$ means the optimal value of $P_{2,4}$ from (27) is negative, but all power values should not be smaller than 0 . Thus, we take $P_{2,4}=0$, and all harvested energy is used in $\tau_{2}$. Then, $P_{2,2}$ can be obtained by (28):

$$
P_{2,2}=\frac{\eta\left|h_{21}\right|^{2}}{1-\eta \sigma_{\mathrm{SI}}^{2}} P_{\max }
$$

and $\tau_{\min }$ can be also derived based on $(17 \mathrm{~d})$,

$$
\tau_{\text {min }}=\frac{C_{1}}{\log _{2}\left(1+\frac{\left|h_{12}\right|^{2}}{N_{0}+\sigma_{\mathrm{RSI}}^{2}} P_{2,2}\right)} .
$$

Then, the initial point of this case can be expressed as:

$$
\begin{aligned}
& \boldsymbol{\tau}^{(1)}=\left[0, \tau_{\min }, 0,1-\tau_{\min }\right]^{T} \\
& \mathbf{P}_{1}^{(1)}=\left[0, P_{\max }, 0,\left(2^{\frac{C_{2}}{1-\tau_{\min }}}-1\right) \frac{N_{0}+\sigma_{\mathrm{RSI}}^{2}}{\left|h_{21}\right|^{2}}\right]^{T} \\
& \mathbf{P}_{2}^{(1)}=\left[0, \frac{\eta\left|h_{21}\right|^{2}}{1-\eta \sigma_{\mathrm{SI}}^{2}} P_{\max }, 0,0\right]^{T} \\
& \alpha_{1}^{(1)}=\frac{2^{\frac{C_{2}}{\tau_{3}+\tau_{4}}}\left\{\left(1-\eta \sigma_{\mathrm{SI}}^{2}\right) N_{0}\right\}^{\frac{\tau_{3}}{\tau_{3}+\tau_{4}}}\left(N_{0}+\sigma_{\mathrm{RSI}}^{2}\right)^{\frac{\tau_{4}}{\tau_{3}+\tau_{4}}}}{\left|h_{21}\right|^{2}} \\
& \alpha_{2}^{(1)}=P_{\max }+\frac{\left(N_{0}+\sigma_{\mathrm{RSI}}^{2}\right)\left(1-\eta \sigma_{\mathrm{SI}}^{2}\right)}{\eta\left|h_{21}\right|^{2}\left|h_{12}\right|^{2}}
\end{aligned}
$$

\subsection{Iterative scheme based on line search method}

As mentioned before, since ACS technique obtains a local minimum, we propose an iterative scheme to derive a value close to global optimum. In proposed scheme, $\boldsymbol{\tau}$ is updated iteratively based on the line search method using gradient of $E_{T}$ about $\boldsymbol{\tau}$ [24]. Then, the $k$ th $\boldsymbol{\tau}$ can be expressed as:

$$
\boldsymbol{\tau}^{(k+1)}=\boldsymbol{\tau}^{(k)}+\beta_{k} \mathbf{p}_{k}
$$

where $\boldsymbol{\tau}^{(k)}$ means updated $\boldsymbol{\tau}$ at the $k$ th iteration, $\mathbf{p}_{k}$ denotes a search direction vector, and $\beta_{k}$ is the magnitude of $\Delta \boldsymbol{\tau}^{(k)}=\boldsymbol{\tau}^{(k+1)}-\boldsymbol{\tau}^{(k)}$. To minimize $E_{T}, \boldsymbol{\tau}$ should be varied from the direction of the maximum partial derivative to that of the minimum partial derivative, because the maximum partial derivative means that the increase of $E_{T}$ is minimum when the duration of the corresponding $\tau$ is decreasing. Thus, $\mathbf{p}_{k}$ can be set as follows:

$$
\mathbf{p}_{k}= \begin{cases}1 & i^{*}=\min _{i} \frac{\partial E_{T}}{\partial \tau_{i}} \\ -1 & j^{*}=\max _{j} \frac{\partial E_{T}}{\partial \tau_{j}} . \\ 0 & \text { otherwise }\end{cases}
$$

Without loss of generality, $P_{1,1}$ can be fixed to $P_{\max }$ before deriving the gradient of $E_{T}$. Since data transmission is not performed during $\tau_{1}$ and the relationship between power and time is linear, minimizing $\tau_{1}$ is efficient in the aspect that it maximizes the available data transmission time. Thus, the gradient of $E_{T}$ over $\tau_{1}$ can be obtained by the condition that the required energy to satisfy $C_{1}$ should not be varied:

$$
\frac{\partial E_{T}}{\partial \tau_{1}}=\left(1-\eta \sigma_{\mathrm{SI}}^{2}\right) P_{\max }+\tau_{2} \frac{\partial P_{1,2}}{\partial \tau_{1}},
$$

where the partial derivatives of $P_{1,3}$ and $P_{1,4}$ can be ignored since these do not affect to $C_{1}$. Then, the partial derivative of $P_{1,2}$ can be written using (11):

$$
\frac{\partial P_{1,2}}{\partial \tau_{1}}=-\frac{P_{\max }}{\tau_{2}} .
$$

Since the magnitude of $P_{1,1}$ is fixed to $P_{\max }, P_{1,2}$ can be represented by the function of the power of $\mathrm{UE}_{2}$ using (11). Then, the gradient of $E_{T}$ about $\boldsymbol{\tau}$ can be expressed using (10) and (11):

$$
\begin{aligned}
& \nabla E_{T}= {\left[-\eta \sigma_{\mathrm{SI}}^{2} P_{\max }, \frac{1-\eta \sigma_{\mathrm{SI}}^{2}}{\eta\left|h_{21}\right|^{2}} P_{2,2}+\tau_{2} \frac{1-\eta \sigma_{\mathrm{SI}}^{2}}{\eta\left|h_{21}\right|^{2}} \frac{\partial P_{2,2}}{\partial \tau_{2}},\right.} \\
&\left(1-\eta \sigma_{\mathrm{SI}}^{2}\right) P_{1,3}+\tau_{3}\left(1-\eta \sigma_{\mathrm{SI}}^{2}\right) \frac{\partial P_{1,3}}{\partial \tau_{3}}, \\
&\left.\frac{1}{\eta\left|h_{21}\right|^{2}} P_{2,4}+P_{1,4}+\tau_{4}\left(\frac{1}{\eta\left|h_{21}\right|^{2}} \frac{\partial P_{2,4}}{\partial \tau_{4}}+\frac{\partial P_{1,4}}{\partial \tau_{4}}\right)\right] .
\end{aligned}
$$

To obtain each partial derivative of (47), we assume that the rates of both UEs are not varied by $\tau$ :

$$
\frac{\partial R_{l}}{\partial \tau_{i}}=0, \quad l=1,2 .
$$

This means that if $\tau_{i}$ is varied, the power corresponding to $\tau_{i}$ is also varied to satisfy target rate. By using (12), (13), (26), (27), and (48), $\nabla E_{T}$ can be expressed in terms of $\alpha_{1}$ and $\alpha_{2}$.

$$
\begin{aligned}
\nabla E_{T} & =\left[-\eta \sigma_{\mathrm{SI}}^{2} P_{\max },\right. \\
& \alpha_{2}-\frac{\left(N_{0}+\sigma_{\mathrm{RSI}}^{2}\right)\left(1-\eta \sigma_{\mathrm{SI}}^{2}\right)}{\eta\left|h_{21}\right|^{2}\left|h_{12}\right|^{2}}-\alpha_{2} \log \left(\frac{\eta\left|h_{21}\right|^{2}\left|h_{12}\right|^{2}}{\left(N_{0}+\sigma_{\mathrm{RSI}}^{2}\right)\left(1-\eta \sigma_{\mathrm{SI}}^{2}\right)} \alpha_{2}\right), \\
& \alpha_{1}-\frac{N_{0}\left(1-\eta \sigma_{\mathrm{SI}}^{2}\right)}{\left|h_{21}\right|^{2}}-\alpha_{1} \log \left(\frac{\left|h_{21}\right|^{2}}{N_{0}\left(1-\eta \sigma_{\mathrm{SI}}^{2}\right)} \alpha_{1}\right), \\
& \alpha_{1}-\frac{N_{0}+\sigma_{\mathrm{RSI}}^{2}}{\left|h_{21}\right|^{2}}-\alpha_{1} \log \left(\frac{\left|h_{21}\right|^{2}}{N_{0}+\sigma_{\mathrm{RSI}}^{2}} \alpha_{1}\right)+ \\
& \left.\alpha_{2}-\frac{N_{0}+\sigma_{\mathrm{RSI}}^{2}}{\eta\left|h_{21}\right|^{2}\left|h_{12}\right|^{2}}-\alpha_{2} \log \left(\frac{\eta\left|h_{21}\right|^{2}\left|h_{12}\right|^{2}}{N_{0}+\sigma_{\mathrm{RSI}}^{2}} \alpha_{2}\right)\right]
\end{aligned}
$$

Since the sum of the phases involved in energy transfer, i.e., $\tau_{1}+\tau_{2}$, should not be smaller than $\tau_{\min }, \mathbf{p}_{k}$ cannot be updated in the direction in which $\tau_{1}+\tau_{2}$ becomes smaller than $\tau_{\min }$. Therefore, the direction finding procedure for updating $\mathbf{p}_{k}$ can be expressed as Table 2 .

The proposed scheme updates $\boldsymbol{\tau}$ considering the variation of $\mathbf{P}_{1}$ and $\mathbf{P}_{2}$ contrary to ACS. $\nabla E_{T}$ can be considered as the difference between the local optimums corresponding to adjacent $\boldsymbol{\tau}$ values. Thus, the energy consumption of 
Table $\mathbf{2}$ The direction finding procedure for updating $\mathbf{p}_{k}$

$$
\begin{array}{ll} 
& \tau_{1}^{(k)}+\tau_{2}^{(k)}=\tau_{\min , j^{*}}=\max _{j} \frac{\partial E_{T}}{\partial \tau_{j}}, \text { where } j^{*} \in\{1,2\} \\
& i^{*}=\min _{i} \frac{\partial E_{T}}{\partial \tau_{i}} \text {, where } i \in\{1,2\}
\end{array}
$$

else

$$
\begin{aligned}
& j^{*}=\max _{j} \frac{\partial E_{T}}{\partial \tau_{j}}, \text { where } j \in[1,4] \\
& i^{*}=\min _{i} \frac{\partial E_{T}}{\partial \tau_{i}}, \text { where } i \in[1,4]
\end{aligned}
$$

end

$k$ th updated $\tau$ can be considered as the lowest local optimum among adjacent local optimums at the $k$ th iteration. Also, the saturated point of the proposed iterative scheme can be explained by using the Hessian matrix of the energy consumption:

$$
H=\left[\begin{array}{cccc}
\frac{\partial^{2} E_{T}}{\partial \tau_{1}^{2}} & 0 & 0 & 0 \\
0 & \frac{\partial^{2} E_{T}}{\partial \tau_{2}^{2}} & 0 & 0 \\
0 & 0 & \frac{\partial^{2} E_{T}}{\partial \tau_{3}^{2}} & 0 \\
0 & 0 & 0 & \frac{\partial^{2} E_{T}}{\partial \tau_{4}^{2}}
\end{array}\right],
$$

and the second-order partial derivatives are given by:

$$
\begin{aligned}
\frac{\partial^{2} E_{T}}{\partial \tau_{1}^{2}}= & 0 \\
\frac{\partial^{2} E_{T}}{\partial \tau_{2}^{2}}= & \frac{\alpha_{2}}{\tau_{2}}\left\{\log \left(\frac{\eta\left|h_{21}\right|^{2}\left|h_{12}\right|^{2}}{\left(N_{0}+\sigma_{\mathrm{RSI}}^{2}\right)\left(1-\eta \sigma_{\mathrm{SI}}^{2}\right)} \alpha_{2}\right)\right\}^{2}, \\
\frac{\partial^{2} E_{T}}{\partial \tau_{3}^{2}}= & \frac{\alpha_{1}}{\tau_{3}}\left\{\log \left(\frac{\left|h_{21}\right|^{2}}{N_{0}\left(1-\eta \sigma_{\mathrm{SI}}^{2}\right)} \alpha_{1}\right)\right\}^{2} \\
\frac{\partial^{2} E_{T}}{\partial \tau_{4}^{2}}= & \frac{\alpha_{1}}{\tau_{4}}\left\{\log \left(\frac{\left|h_{21}\right|^{2}}{\left(N_{0}+\sigma_{\mathrm{RSI}}^{2}\right)} \alpha_{1}\right)\right\}^{2} \\
& +\frac{\alpha_{2}}{\tau_{4}}\left\{\log \left(\frac{\eta\left|h_{21}\right|^{2}\left|h_{12}\right|^{2}}{\left(N_{0}+\sigma_{\mathrm{RSI}}^{2}\right)} \alpha_{2}\right)\right\}^{2} .
\end{aligned}
$$

Since $\alpha_{1}>0, \alpha_{2}>0$, and $\tau_{i} \geq 0$, each second-partial derivative is not less than 0 . This means that Hessian matrix about $\boldsymbol{\tau}$ is positive semi-definite even if $\mathbf{P}_{1}$ and $\mathbf{P}_{2}$ are variables about $\boldsymbol{\tau}$. Therefore, the energy consumption according to local optimum sets which are obtained by the proposed iterative scheme is convex about $\boldsymbol{\tau}$, the solution of the proposed scheme can saturate to the value close to global optimum.

Next, $\beta_{k}$ should be derived for updating $\tau$. $\beta_{k}$ decides the convergence gap $\epsilon$ and the number of iterations up to saturation. $E_{T}$ can be modified the approximated objective function as Taylor series.

$$
\begin{aligned}
& E_{T}(\tau) \approx \\
& E_{T}\left(\tau^{(k)}\right)+\nabla E_{T}\left(\tau^{(k)}\right)\left(\tau-\tau^{(k)}\right) \\
& \quad+\frac{1}{2}\left(\tau-\tau^{(k)}\right)^{T} \nabla^{2} E_{T}\left(\tau^{(k)}\right)\left(\tau-\tau^{(k)}\right),
\end{aligned}
$$

where $\nabla^{2} E_{T}\left(\tau^{(k)}\right)$ is Hessian matrix of $E_{T}$ at $\tau^{(k)}$ and the higher order terms are ignored. Then, $E_{T}\left(\tau^{(k+1)}\right)$ is:

$$
\begin{aligned}
E_{T}\left(\tau^{(k)}+\beta_{k} \mathbf{p}_{k}\right) \approx & E_{T}\left(\tau^{(k)}\right)+\nabla E_{T}\left(\tau^{(k)}\right) \beta_{k} \mathbf{p}_{k} \\
& +\frac{1}{2} \beta_{k} \mathbf{p}_{k}^{T} \nabla^{2} E_{T}\left(\tau^{(k)}\right) \beta_{k} \mathbf{p}_{k} .
\end{aligned}
$$

Since $\mathbf{p}_{k}$ is the direction toward to decreasing the total energy consumption and $E_{T}\left(\tau^{(k+1)}\right) \leq E_{T}\left(\tau^{(k)}\right)$,

$$
\begin{aligned}
\nabla E_{T, \min }\left(\tau^{(k)}\right) \beta_{k} & -\nabla E_{T, \max }\left(\tau^{(k)}\right) \beta_{k} \\
+ & \frac{1}{2} \beta_{k}^{2}\left(\nabla^{2} E_{T, \min }+\nabla^{2} E_{T, \max }\right) \leq 0,
\end{aligned}
$$

where the direction of maximum partial derivative is -1 , that of minimum partial derivative is 1 , and others are $0 . \nabla E_{T, \min }\left(\tau^{(k)}\right)$ and $\nabla^{2} E_{T, \min }\left(\tau^{(k)}\right)$ mean partial and second partial derivatives at $\tau^{(k)}$ corresponding to $i^{*}$, respectively, and $\nabla E_{T, \max }\left(\tau^{(k)}\right)$ and $\nabla^{2} E_{T, \max }\left(\tau^{(k)}\right)$ denote partial and second partial derivatives at $\tau^{(k)}$ corresponding to $j^{*}$, respectively. Then the scaling factor $\beta_{k}$ can be expressed as:

$$
\beta_{k} \leq \frac{2\left(\nabla E_{T, \max }\left(\tau^{(k)}\right)-\nabla E_{T, \min }\left(\tau^{(k)}\right)\right)}{\nabla^{2} E_{T, \max }\left(\tau^{(k)}\right)+\nabla^{2} E_{T, \min }\left(\tau^{(k)}\right)} .
$$

Since all components of $\tau$ cannot be smaller than 0 , the upper bound of $\beta_{k}$ should be defined as:

$\beta_{k}=\min \left(\gamma \times \tau_{j^{*}}, \frac{2\left(\nabla E_{T, \max }\left(\tau^{(k)}\right)-\nabla E_{T, \min }\left(\tau^{(k)}\right)\right)}{\nabla^{2} E_{T, \max }\left(\tau^{(k)}\right)+\nabla^{2} E_{T, \min }\left(\tau^{(k)}\right)}\right)$,

where $\gamma \times \tau_{j *}$ is an upper bound to prevent $\beta_{k}$ from becoming larger than $\tau_{j *}$. The proposed optimization scheme can obtain a value close to global optimum, since the Hessian matrix of $E_{T}$ about $\tau$ is positive semi-definite.

\section{Simulation results}

In this section, we discuss the pros and cons of the proposed system compared with the conventional SWIPT systems. We set the path loss exponent as $2, N_{0}=$ $-70[\mathrm{dBm}], P_{\max }=23[\mathrm{dBm}]$, and $\eta=0.5[22,25,26]$. We set $\epsilon=10^{-2}, \gamma=0.1$, and $k_{\max }=1000$ for the iterative scheme. 
Figure 2 shows the convergence of the proposed scheme compared to ACS where $\sigma_{\text {SIC }}^{2}=-100[\mathrm{~dB}]$. The values of the exhaustive search are derived by comparing all local minimums when $\boldsymbol{\tau}$ is fixed. In exhaustive search, the difference of each fixed $\boldsymbol{\tau}$ between adjacent local minimums is set 0.05 . This means that the difference between the global optimum point and the point of the exhaustive search is smaller than 0.05 , i.e., $\left|\tau^{\mathrm{opt}}-\tau^{\mathrm{exh}}\right| \leq 0.05$, where $\tau^{\text {opt }}$ is the global optimum point of $\tau$ and $\tau^{\text {exh }}$ denotes the nearest point to $\tau^{\text {opt }}$ among local minimums of the exhaustive search. In ACS scheme, linear programming is used to derived local minimum when $\mathbf{P}_{1}$ and $\mathbf{P}_{2}$ are fixed, and the KKT conditions are used to find local minimum when $\tau$ is fixed. It is shown that the performance of the proposed iterative optimization scheme is almost the same as the performance of the exhaustive search. In contrast to the proposed scheme, the results of ACS cannot obtain the value similar to exhaustive search since ACS technique cannot assure global optimum. Also, we can see that the performance of ACS depends on the initial points. The performance of ACS with the proposed initial point is similar to that of the proposed scheme in low $\sigma_{\mathrm{SI}}^{2}$ region because the efficiency of S-ER is trivial in low $\sigma_{\mathrm{SI}}^{2}$ region. This means that the initial point obtained in Section 3.2 is close to the optimal value since simultaneous transmission is more efficient than S-ER. However, the optimal point is far from the initial point obtained by Section 3.2 when $\sigma_{\mathrm{SI}}^{2}$ is large since the performance difference between the exhaustive search and
ACS with the proposed initial point becomes large for larger $\sigma_{\mathrm{SI}}^{2}$.

Figure 3 shows the minimum energy consumption of various systems according to $\sigma_{\mathrm{SI}}^{2}$. Half-duplex (HD) SWIPT system can be considered as a system with $\tau_{4}=$ $0, P_{1,2}=0$, and $\sigma_{\mathrm{RSI}}^{2}=0$ in Fig 1 . Also, FD SWIPT system can be treated as a system considering only $\tau_{2}$ and $\tau_{4}$ in Fig 1 . It can be seen that the proposed system and FD system have almost the same energy consumption while HD system has the worst performance in low $\sigma_{\mathrm{SI}}^{2}$ region, because the effectivenesses of S-ER and residual self-interference are trivial. This means that most of the transmission time is used for simultaneous data transmission in the proposed system, and the time allocation of the proposed system is almost the same with FD system. Therefore, the energy consumption of FD system is similar to that of the proposed system in low $\sigma_{\mathrm{SI}}^{2}$ region. In the case of HD system, however, simultaneous data transmission time is not assigned. This causes an increase of transmit power to achieve target rates of both users; thus, the energy consumption of HD system has the largest value. However, the performance gap between FD system and HD system is decreased as $\sigma_{\mathrm{SI}}^{2}$ becomes larger. It is because the efficiency of S-ER increases as $\sigma_{\mathrm{SI}}^{2}$ becomes larger in HD system, and the performance loss due to residual self-interference occurs in FD system. In HD system, the reduction rate of the energy consumption is the largest since S-ER is operated in all transmission phase. On the other hand, the reduction rate of the energy

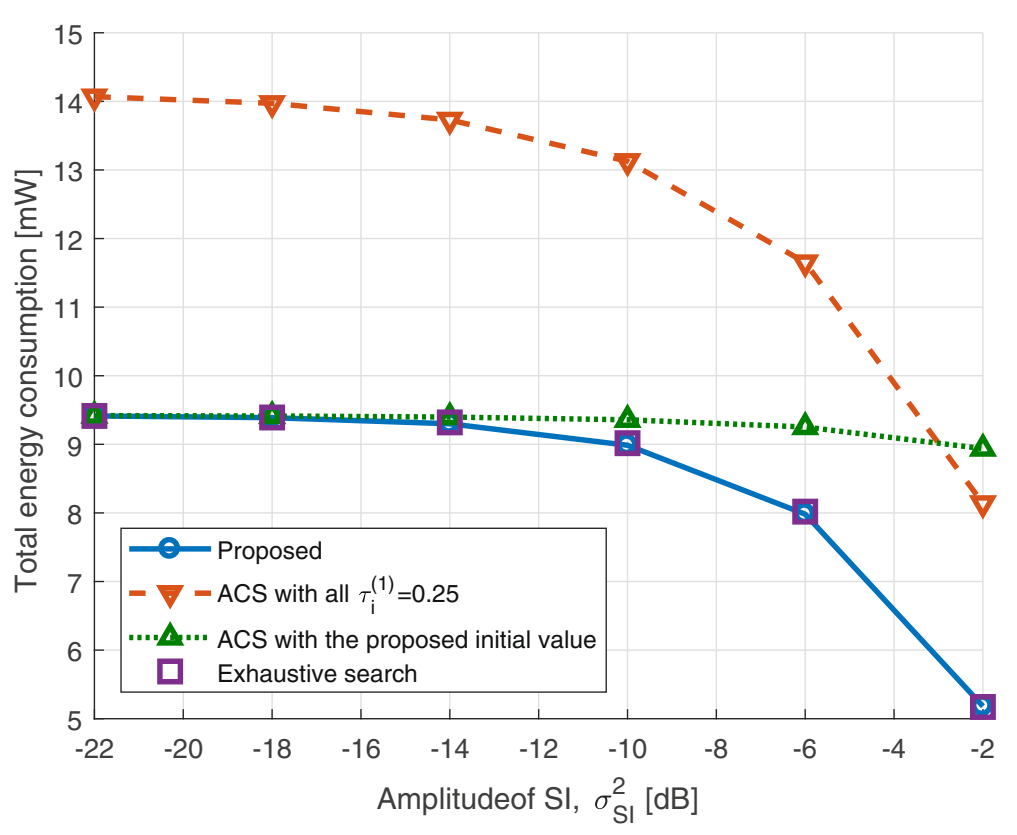

Fig. 2 Convergence of the proposed scheme compared to ACS. $C_{1}=C_{2}=1[\mathrm{bps} / \mathrm{Hz}], D=10[\mathrm{~m}]$ 


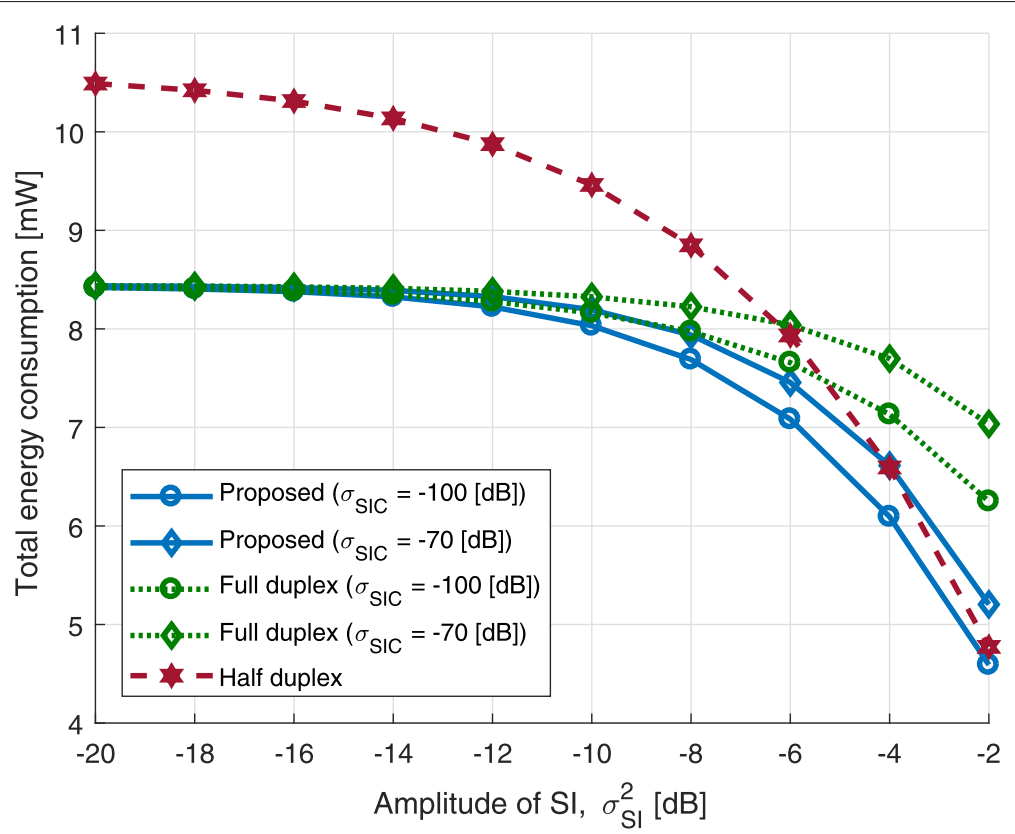

Fig. 3 Total energy consumptions of various systems according to $\sigma_{\mathrm{S} \mid}^{2} \cdot C_{1}=C_{2}=1[\mathrm{bps} / \mathrm{Hz}], D=10[\mathrm{~m}]$

consumption of FD system is the smallest because S-ER is only implemented during one phase. Also, the effect of residual self-interference cannot be ignored. Thus, the energy consumption of HD system is less than that of FD system if the magnitude of $\sigma_{\mathrm{SI}}^{2}$ is larger than $-5[\mathrm{~dB}]$ and $-6[\mathrm{~dB}]$ when $\sigma_{\mathrm{SIC}}^{2}=-100[\mathrm{~dB}]$ and $\sigma_{\mathrm{SIC}}^{2}=-70[\mathrm{~dB}]$, respectively. Similarly, the performance gap between the proposed system and FD system increases as the magnitude of $\sigma_{\mathrm{SI}}^{2}$ grows. In the proposed system, most of the transmission time is assigned to simultaneous data transmission phase when the magnitude of $\sigma_{\mathrm{SI}}^{2}$ is small since the efficiency of simultaneous data transmission is higher than that of S-ER. However, time allocation to S-ER phase is increased as the efficiency of S-ER becomes higher, which makes the energy consumption of the proposed system more efficient than that of FD system. In the case of $\sigma_{\mathrm{SIC}}^{2}=-70[\mathrm{~dB}]$, the performance of the proposed system is lower than that of HD system because the power of the residual self-interference is fixed to the upper bound level. Ideally, the time allocation to $\tau_{1}$ is increased, and most of the harvesting energy of $\mathrm{UE}_{2}$ is transferred during $\tau_{1}$ as $\sigma_{\mathrm{SI}}^{2}$ become larger. This means that the transmission power of $\mathrm{UE}_{1}$ during $\tau_{2}$ becomes smaller. However, since the power of the residual self-interference is not varied keeping upper bound value, the performance of the proposed system is lower than that of HD system in high $\sigma_{\text {SI }}^{2}$ region if sufficient self-interference cancelation is not conducted.

Next, we discuss the effect of $P_{\max }$, and the simulation result is shown in Fig. 4. It can be seen that the energy consumption of HD system is decreased as $P_{\max }$ becomes larger because $\mathrm{HD}$ system has the phase where only energy transmission is operated. Since the length of this phase is reduced as $P_{\max }$ becomes larger, available data transmission time can be increased, and it decreases the required energy for data transmission. Therefore, the energy consumptions of HD system decreases as $P_{\max }$ becomes larger. This tendency is also shown in the proposed system with $\sigma_{\mathrm{SIC}}=$ $-100[\mathrm{~dB}]$. However, the energy consumption of FD system where $\sigma_{\mathrm{SIC}}=-100[\mathrm{~dB}]$ does not change according to $P_{\max }$ because FD system does not have an energy transfer only phase. Also, the length of the transmission time corresponding to each phase is dependent to data transmission rather than energy transfer. Thus, decreasing energy transmission time by increasing $P_{\max }$ does not affect the variation of the length of each phase in FD system. In the case of $\sigma_{\text {SIC }}^{2}=-70[\mathrm{~dB}]$, both energy consumption of the proposed system and FD system are increased as $P_{\max }$ becomes larger. Since the variance of the residual self-interference is affected by $P_{\max }$, the performance degradation occurs in both system if sufficient self-interference cancelation is not conducted.

Figure 5 shows the total energy consumption according to target rate where $\sigma_{\mathrm{SI}}^{2}=-2[\mathrm{~dB}]$. It can be seen that the increase of the energy consumption according to target rate is largest in HD system, because simultaneous data transmission phase does not exist in HD system. Thus, the energy consumption of HD system is less than 


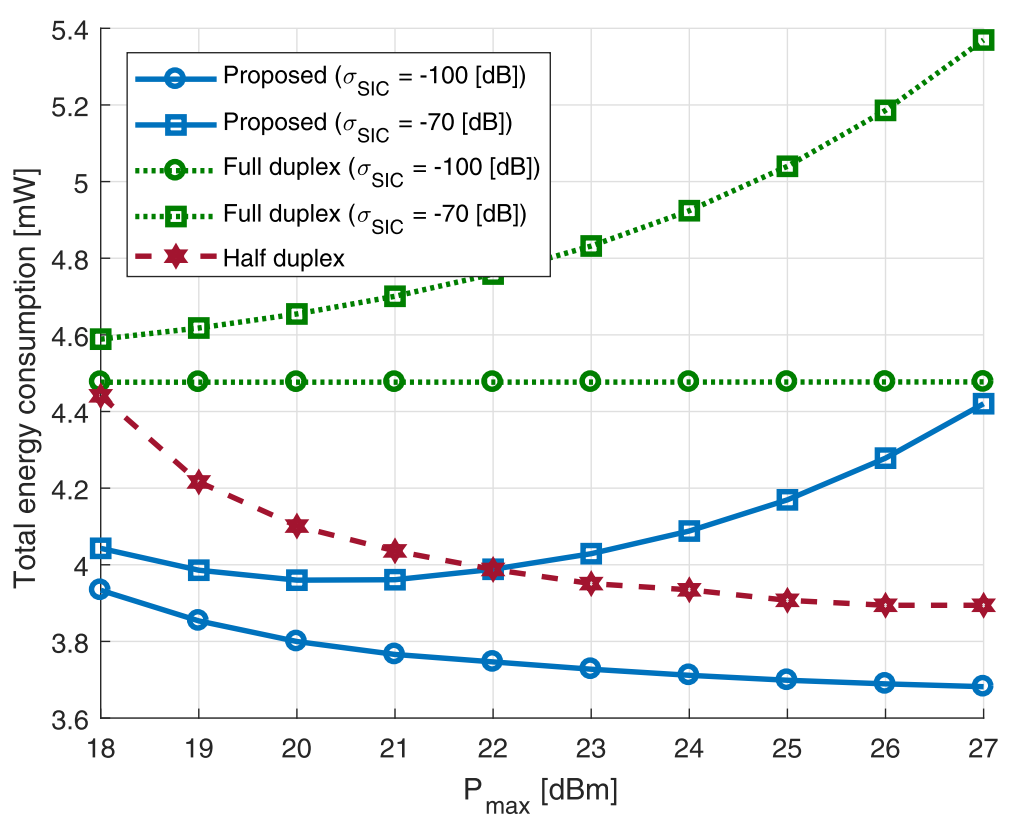

Fig. 4 Total energy consumptions of various systems according to $P_{\max } C_{1}=C_{2}=1[\mathrm{bps} / \mathrm{Hz}], \sigma_{\mathrm{Sl}}^{2}=-4[\mathrm{~dB}]$, and $D=10[\mathrm{~m}]$

that of FD system when target rate is $2.5[\mathrm{bps} / \mathrm{Hz}]$, but the energy consumption of HD system is greater than that of FD system when target rate is larger than 4 [ bps/Hz]. In low target rate region, S-ER phase is more efficient than simultaneous transmission phase since $\sigma_{\mathrm{SI}}^{2}$ is large.
However, simultaneous data transmission is more efficient than S-ER because energy consumption increases exponentially as target rate become higher. Thus, the performance reversal between HD and FD systems occurs. On the other hand, the proposed system has the lowest

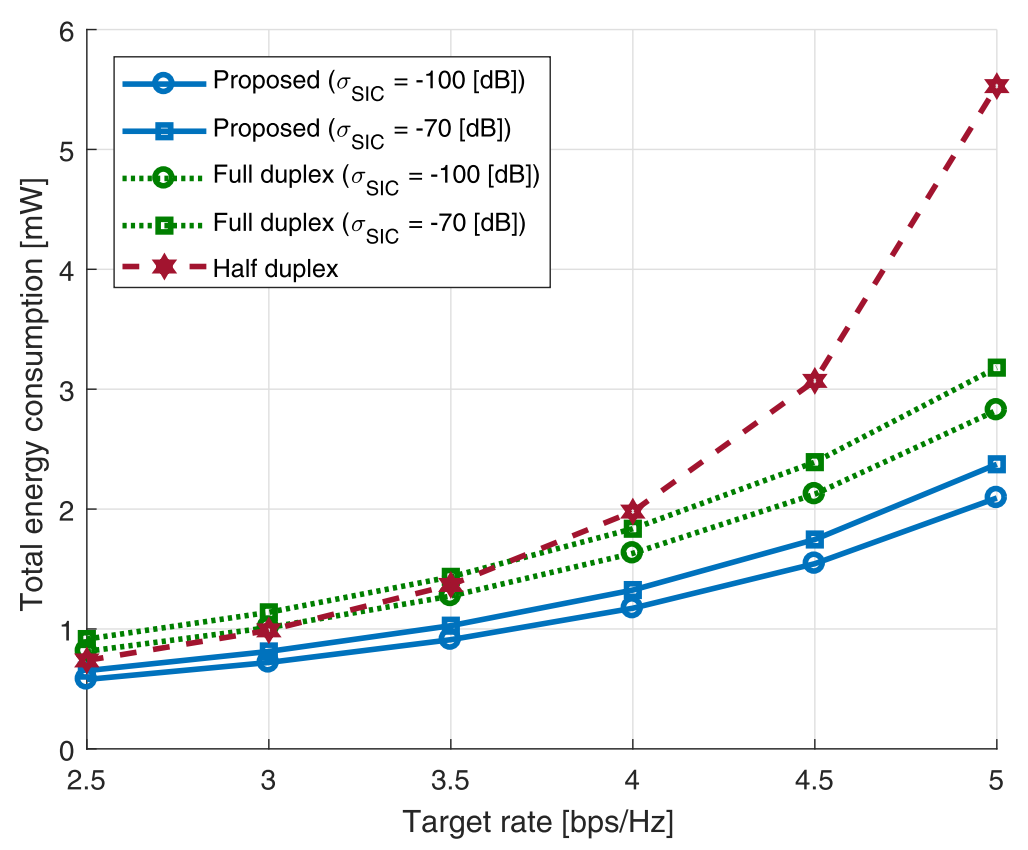

Fig. 5 Total energy consumptions of various systems according to target rate. $\sigma_{S \mid}^{2}=-2[\mathrm{~dB}], D=5[\mathrm{~m}]$ 
energy consumption in all target rate region, because the proposed system can efficiently allocate the time using both S-ER phase and simultaneous data transmission phase.

\section{Conclusions}

In this paper, we propose the TS-based FD SWIPT system in D2D communication system to minimize total energy consumption where S-ER is implemented at both users. Harvesting SI is more efficient than simultaneous data transmission when the magnitude of SI is sufficiently large. Thus, S-ER operating phases are combined in the proposed system, and this enables efficient time and power allocation. To minimize total energy consumption, we optimize transmission time and power corresponding to each phase under target rate constraints. Also, in contrast to the previous power minimization problems, we limit maximum transmission power of users to reflect practical limitation. First, we show the optimization problem of the proposed system is bi-convex problem, and the optimal power allocation scheme is proposed when the transmission time of each duration is fixed. Though ACS can be applied to solve the conventional bi-convex problem, it can give only a local optimum solution. Thus, we proposed the iterative algorithm to derive a better solution based on the line search method. Numerical results showed the proposed system gives better performance compared with the conventional SWIPT systems. However, since the proposed system is limited to single D2D pair, it needs to expand to general multiple D2D pairs system by considering the different interference patterns from each D2D pair.

\section{Methods/Experimental}

The purpose of this study is to minimize the system consumption energy. The system consists of two FD users where imperfect self-interference cancelation is assumed. The channels between each user are assumed as Rayleigh fading channel, and the self-interference channel of each user is assumed as random variable with static amplitude and random phase. The simulation results of the conventional schemes and exhaustive search are derived by comparing all local minimum when $\tau$ is fixed, and each local minimum is derived by using optimal power allocation scheme which is introduced in Section 3.1. HD SWIPT system can be considered as the proposed system with $\tau_{4}=0, P_{1,2}=0$, and $\sigma_{\mathrm{RSI}}^{2}=0$. Also, FD SWIPT system can be treated as a system set to $\tau_{1}=0$ and $\tau_{3}=0$ in the proposed system model. In the process of finding each local minimum, the difference of each fixed $\boldsymbol{\tau}$ between adjacent local minimums is set to 0.05 . This means that the difference between the global optimum point and the point of the exhaustive search is smaller than 0.05 , i.e., $\left|\tau^{\text {opt }}-\tau^{\text {exh }}\right| \leq 0.05$. Since the feasible set may not exist due to the limitation of maximum transmission power, we take the system parameters which make the feasibility of all schemes close to 1 . Also, if any of the entire systems has no feasible set, the results of its instantaneous channel model are excluded from Monte Carlo simulations.

\begin{abstract}
Abbreviations
ACS: Alternative convex search; DL: Downlink; D2D: Device-to-device; EH: Energy harvesting; FD: Full duplex; HD: Half duplex; ID: Information detection; IOT: Internet of things; MIMO: Multiple-input multiple-output; MISO: Multiple-input single-output; PS: Power splitting; P2P: Point-to-point; S-ER: Self-energy recycling; SWIPT: Simultaneous wireless information and power transfer; TS: Time switching; UE: User equipment
\end{abstract}

Funding

This study was supported by the National Research Foundation of Korea (NRF-2015R1D1A1A01058644).

\section{Availability of data and materials}

Data sharing is not applicable to this article as no datasets were generated or analyzed during the current study.

\section{Authors' contributions}

HN has contributed $70 \%$ of the work, and the rest is contributed by CL. Both authors read and approved the final manuscript.

\section{Competing interests}

The authors declare that they have no competing interests.

\section{Publisher's Note}

Springer Nature remains neutral with regard to jurisdictional claims in published maps and institutional affiliations.

\section{Author details}

${ }^{1}$ The Department of Electrical and Electronic Engineering, Yonsei University, C418, 03722 Seoul, South Korea. ${ }^{2}$ The Department of Electrical and Electronic Engineering, Yonsei University, C527, 03722 Seoul, South Korea.

Received: 11 April 2018 Accepted: 18 September 2018

Published online: 25 October 2018

\section{References}

1. L.R. Varshney, in IEEE International Symposium on Information Theory - 573 Proceedings. Transporting information and energy simultaneously (IEEE, Toronto, 2008), pp. 1612-1616

2. L. Liu, R. Zhang, K.C. Chua, Wireless information transfer with opportunistic energy harvesting. IEEE Trans. Wirel. Commun. 12(1), 288-300 (2013)

3. D.W.K. Ng, E.S. Lo, R. Schober, Wireless information and power transfer: energy efficiency optimization in OFDMA systems. IEEE Trans. Wirel. Commun. 12(12), 6352-6370 (2013)

4. Q. Shi, L. Liu, W. Xu, R. Zhang, Joint transmit beamforming and receive power splitting for MISO SWIPT systems. IEEE Trans. Wirel. Commun. 13(6), 3269-3280 (2014)

5. X. Zhou, R. Zhang, C.K. Ho, Wireless information and power transfer in multiuser OFDM systems. IEEE Trans. Wirel. Commun. 13(4), 2282-2294 (2014)

6. M. Maso, C.F. Liu, C.H. Lee, T.Q.S. Quek, L.S. Cardoso, Energy-recycling full-duplex radios for next-generation networks. IEEE J. Sel. Areas Commun. 33(12), 2948-2962 (2015)

7. M. Mohammadi, H.A. Suraweera, G. Zheng, C. Zhong, I. Krikidis, in IEEE Workshop on Signal Processing Advances in Wireless Communications, SPAWC. Full-duplex MIMO relaying powered by wireless energy transfer, vol. 2015-Augus (IEEE, Stockholm, 2015), pp. 296-300

8. Y. Zeng, R. Zhang, Full-duplex wireless-powered relay with self-energy recycling. IEEE Wirel. Commun. Lett. 4(2), 201-204 (2015) 
9. Z. Hu, C. Yuan, F. Zhu, F. Gao, Weighted sum transmit power minimization for full-duplex system with SWIPT and self-energy recycling. IEEE Access. 4, 4874-4881 (2016)

10. D. Wang, R. Zhang, X. Cheng, L. Yang, Capacity-enhancing full-duplex relay networks based on power-splitting (PS-)SWIPT. IEEE Trans. Veh. Technol. 66(6), 5446-5450 (2017)

11. X. Zhou, R. Zhang, C.K. Ho, Wireless information and power transfer: architecture design and rate-energy tradeoff. IEEE Trans. Commun. 61(11), 4754-4767 (2013)

12. Y. Wu, Y. Liu, Q. Xue, S. Li, C. Yu, Analytical design method of multiway dual-band planar power dividers with arbitrary power division. IEEE Trans. Microw. Theory Tech. 58(12), 3832-3841 (2010)

13. Y. Zeng, R. Zhang, Full-duplex wireless-powered relay with self-energy recycling. IEEE Wirel. Commun. Lett. 4(2), 201-204 (2015)

14. Z. Ding, S. Hu, Q. Ni, Beamforming optimisation in energy harvesting cooperative full-duplex networks with self-energy recycling protocol. IET Commun. 10(7), 848-853 (2016)

15. D. Hwang, K.C. Hwang, D.I. Kim, T.J. Lee, Self-energy recycling for RF powered multi-antenna relay channels. IEEE Trans. Wirel. Commun. 16(2), 812-824 (2017)

16. Y. Liao, K. Bian, L. Song, Z. Han, Full-duplex MAC protocol design and analysis. IEEE Commun. Lett. 19(7), 1185-1188 (2015)

17. T. Riihonen, S. Werner, R. Wichman, Mitigation of loopback self-interference in full-duplex MIMO relays. IEEE Trans. Signal Process. 59(12), 5983-5993 (2011)

18. M. Jain, J.I. Choi, T. Kim, D. Bharadia, S. Seth, K. Srinivasan, P. Levis, S. Katti, P. Sinha, in Proceedings of the 17th Annual International Conference on Mobile Computing and Networking. Practical, real-time, full duplex wireless, MobiCom '11 (ACM, New York, 2011), pp. 301-312

19. D.W.K. Ng, E.S. Lo, R. Schober, Dynamic resource allocation in MIMO-OFDMA systems with full-duplex and hybrid relaying. IEEE Trans. Commun. 60(5), 1291-1304 (2012)

20. D. Bharadia, E. McMilin, S. Katti, Full duplex radios. SIGCOMM Comput. Commun. Rev. 43(4), 375-386 (2013)

21. L.J. Rodríguez, N.H. Tran, Performance of full-duplex AF relaying in the presence of residual self-interference. IEEE J. Sel. Areas Commun. 32(9), 1752-1764 (2014)

22. Y. Chang, H. Chen, F. Zhao, Energy efficiency maximization of full-duplex two-way relay-assisted device-to-device communications underlaying cellular networks. EURASIP J. Wirel. Commun. Netw. 2016(1), 222-224 (2016)

23. J. Gorski, F. Pfeuffer, K. Klamroth, Biconvex sets and optimization with biconvex functions: a survey and extensions. Math. Methods Oper. Res. 66(3), 373-407 (2007)

24. E.K.P. Chong, S.H. Zak, An Introduction to Optimization, 3rd. (Wiley-Interscience, New Jersey, 2008)

25. S. Ali, N. Rajatheva, M. Latva-aho, in 2014 European Conference on Networks and Communications (EuCNC). Full duplex device-to-device communication in cellular networks (IEEE, Bologna, 2014), pp. 1-5

26. Z. Zhou, M. Dong, K. Ota, J. Wu, T. Sato, Energy efficiency and spectral efficiency tradeoff in device-to-device (D2D) communications. IEEE Wirel. Commun. Lett. 3(5), 485-488 (2014)

\section{Submit your manuscript to a SpringerOpen ${ }^{\circ}$ journal and benefit from:}

- Convenient online submission

- Rigorous peer review

- Open access: articles freely available online

- High visibility within the field

- Retaining the copyright to your article

Submit your next manuscript at $>$ springeropen.com 